\title{
Dynamic Window-based Adaptive Median Filter Alogorithm
}

\author{
Shi Junyan ${ }^{1,2, *}$ and Liu Shudan ${ }^{1,2}$ \\ ${ }^{I}$ Computer Application Engineering Research Center of Xuchang City, Xuchang, Henan, 461000, P.R. China; \\ ${ }^{2}$ Xuchang Vocational and Technical College, Xuchang, Henan, 461000, P.R. China
}

\begin{abstract}
In response to the flaw that the median filtering algorithm has a poor handling capacity to high-density and fine texture noise, a Dynamic window-based adaptive median filter algorithm is proposed. According to the associated level between noise-point information and the surrounding, the new algorithm adjust, the Noise point filter value, which can get a better deal with the details of the images. The adaptive strategies strengthen the performance of the filtering de-noising algorithm, which is good at dealing the high-density noise. Through simulation analysis, the new algorithm is better than other algorithms.
\end{abstract}

Keywords: Adaptive strategy, dynamic window, filter algorithm, noise detection, salt-and-pepper noise.

\section{INTRODUCTION}

In the coding and transmission of images, it often needs salt-and-pepper noise (i.e. impulse noise) to filter processing for image [1]. Since of median filter has mapping relationship with the input signal sequence and has good effect in removing impulse noise, many scholars have researched on median filtering technology, made tremendous of improvement algorithms. Such as weighted median filtering method (WM) [2], center weights median filter (CWM) [3], three state median filter (TSM) [4], fuzzy multi-polar median filtering method [6] etc., and improvement strategies based on several methods described above [7]. Document [8] introduced an improved adaptive median filtering method (AM), made a good filtering effect, but for high density image noise and delicate texture image processing power is not too strong [9]. Based on AM algorithm framework, by analyzing the image noise information, a new adaptive median filter based on noise detection is proposed in this paper to overcome high density noise and more details of image noise are not ideal. Experimental results show that the new algorithm is good for detail-rich images and high density image filtering effect of noise, effectively improves the image of the peak signal noise ratio, the de-noising better than other methods.

\section{MEDIAN FILTER INTRODUCTION}

As early as 1974, Turkey proposed one-dimensional median filter, then some scholars have successfully extended to two-dimensional image. Standard median filter (SM) is divided into sub-image using a sliding window, and then the sub-image is a two-dimensional median filter. In the process of filtering, the window size can be set to different values; the window is typically a square $3 * 3$. The specific method is pixel on the current window, which is sorted gray value, taking the median value as the gray value of the current pixel. Due to the lack of a mechanism to judge whether the pixel is affected by noise, using this method is required when a filtering operation on all pixels, to a certain extent, will damage the edge detail information of image.

\section{NOISE POINT DETECTION}

The image pixel from the effects of salt-and-pepper noise, the gray value is maximum or minimum [9]. For this feature, before the de-noising process, pixels can be divided into non-noise pixel point, the noise point and image detail point, in order to achieve the purpose of reducing system overhead and to protect non-noise points. Detection of noise points through two levels, the global detection and local detection, to determine, as described below.

\subsection{Global Detection}

In the affected image by salt-and-pepper noise, the gray value distribution of noise points in image gray value maximum or minimum terminal end. If gray value of certain point in the image is the middle of extremes, you can conclude that the current point is not noised; you do not need to de-noise. For extreme values in pixels, it cannot determine whether it is a noise point.

Setting the maximum value of the image gray value Gmax, the minimum value Gmin, the current pixel gray value $\mathrm{G}$, if it satisfies the formula (1), the current pixel cannot be described by the noise pollution, does not perform the filtering operation, where $\mathrm{T}$ is the set threshold value.

$$
|G-G \min |>T \text { 并且 }|G-G \max |>T
$$

\subsection{Local Detection}

The purpose of local detection is to determine the local gray value at the extreme point whether noise points. In Fig. (1), the distribution of a large number of black and white stripes, namely the existence of a large number of extreme 




Fig. (1). There are a lot extremes barb figure.

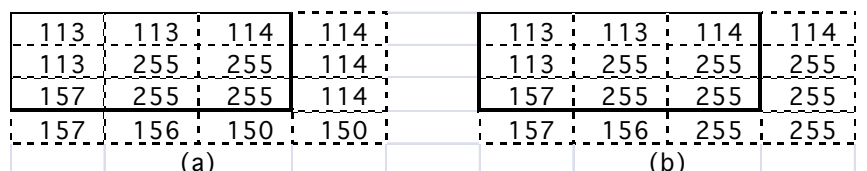

Fig. (2). Image gray value.

values of pixels in the original image. For this kind of characteristic image, due to WM, CWM, TSM and other majority of median filtering method because the gray value of the non-noise pixels extreme misjudgment as noise points, treatment result is not very ideal. How to ensure the nonextreme points are not noise filter, or not misplaced with larger difference with the surrounding pixels after filter, it requires full information about the pixels surrounding the binding analysis. Shown in Fig. (2), the $3 * 3$ filtering window, for table (a), because the larger difference of the gray value between the center point in window and adjacent points, the possibility of noise points is larger; and for table (b), since the difference is small $(=0)$, the possibility of nonnoise points is larger.

\subsection{Noise Detection Algorithm}

From the above analysis, can be summarized as a complete noise detection algorithm.

Noise detection algorithm

Input: global image of the maximum value Gmax, the minimum value Gmin, the filter window is a maximum value Wmax, the pixel P $(a, b)$ and the gray value $G$, the threshold value $\mathrm{T}$

Input: the image global maximum value Gmax, minimum value Gmin, the maximum value of filter window is Wmax, pixel $P(a, b)$ and the gray value $G$, the threshold value $T$

Output: the determination of pixel $\mathrm{P}(\mathrm{a}, \mathrm{b})$.

1. If meet $\mid$ G-Gmin $\mid>\mathrm{T}$ and $\mid$ G-Gmax $\mid>\mathrm{T}$, then turn 6;

2. $P$ as the center sets the window size $\mathrm{w}$ for the filter window 3 ;

3. Calculate the standard median filter results in a window, denoted as SM; if meet $\mathrm{Gmin}<\mathrm{SM}<\mathrm{Gmax}$, then go to 5 ;

4. Sets the window size expands to $w+2$; if $w>W$ max, then goes to 5 , otherwise goes to 3 ;

5. For the current filtering window, calculate pixel $\mathrm{P}$ and another $\mathrm{w} * \mathrm{w}-1$ pixel gray value difference of mean Gmean; if Gmean $<\mathrm{T} 1$, turn 6, otherwise turn 7;
6. The point $\mathrm{P}$ is non-noise points.

7. The point $P$ is the noise points.

\subsection{Adaptive Window Strategy}

In the course of detection of noise points, the size of the filter window has a very large impact on the filtering algorithm: if the value of the window is small, can effectively protect the image details, but the de-noising effect is relatively weak; on the contrary, the filter go noise effect is strong, and the filtered image blurring will increase.

Fig. (3) shows the noise of a local region in the image gray value matrix, when a large noise in the image density, a smaller window is not guaranteed Gmean shows the relationship between the right edge of the window and the center value of the other pixel points. Such as (a), the $3 * 3$ window contains a noise six points, but extremum are maximum value, the difference between the mean and the center of the window of the other eight pixels is still 20, and the window center at this time is determined to be non-noise points; and when the window is expanded, although the increase in noise point, but the ratio of the maximum value and the minimum value changes, thereby reducing the impact of noise on Gmean the extreme points, the obtained Gmean also correctly reflects the center of the window is a noise point, (b) it can be seen when taking $5 * 5$ window, Gmean calculated to be greater than $\mathrm{T} 1$, the center of the window can be determined as a noise point.

\begin{tabular}{|c|c|c|c|c|c|c|}
\hline & & 180 & 255 & 177 & 255 & 187 \\
\hline 255 & $2255 \quad 187$ & 0 & 255 & 255 & 187 & 0 \\
\hline 168 & 255 & 167 & 168 & 255 & -255 & $\begin{array}{r}255 \\
-\end{array}$ \\
\hline 160 & 255 & 167. & 160 & 255 & 255 & 255 \\
\hline & & 0 & 147 & 147 & 255 & 0 \\
\hline
\end{tabular}

Fig. (3). Image noise density of 0.6 local information.

Principles for selecting the filter window size is to point to the influence of noise in the window Gmean minimum. Used here SM value of the standard median filter is compared. If the value of SM is between the minimum value and the maximum value, it can be described in more homogeneous distribution of maxima and minima of the values of the filtering window.

The role of adaptive filtering window is not only reflected in the noise point judgment, to filter out the noise of operation, adaptive window has also an important role, this content will be described in detail below.

\section{FILTER OUT NOISE POINTS}

Adaptive window policy can also be more accurate to distinguish the noise points and image details point to better protect the image details and better able to de-noising performance tuning of the filter, effectively compensate for the general filter for containing high less than the density of the noise on the image processing.

Fig. (4) shows the noise density of 0.2 lena diagram using AM filter de-noising situation in which (a) is the original image, (b) a $3 * 3$ window, (c) a $5 * 5$ window, (d) using $9 *$ 9 window. As can be seen, the first point of the filtered noise cannot be completely filtered out at a $3 * 3$ window, and after a $9 * 9$ window, although the noise has been filtered 
out, but the image relative to the original image have been greatly blurred. Size can be seen, the window has important implications for the filter de-noising effect. When the filter window is smaller, richer image detail retained, but poor denoising performance; and when the window increases, denoising performance has improved significantly, but will also be blurred image details.

When an image contains a high noise density, the window size is more apparent. 5, when the noise density of 0.8 , the $3 * 3$ window through the value SM and the right and left values are all ortho to the point of noise, then the filtering operation will not be of any change in its gray value, the filter has lost its meaning. At this point the window size needs to obtain more image information to compensate for the effects of noise caused.

\begin{tabular}{|c|c|c|c|c|c|c|c|}
\hline & & & 0 & 167 & 0 & 255 & 255 \\
\hline 255 & 255 & 255 \\
\hline 134 & 255 & 255 \\
\hline 255 & 255 & 134 \\
\hline & & & 255 & 255 & 255 & 255 & 167 \\
\hline (a) & & 255 & 134 & 255 & 255 & 0 \\
\hline 134 & 255 & 255 & 134 & 0 \\
\hline 0 & 0 & 0 & 255 & 0 \\
\hline
\end{tabular}

Fig. (4). lena figure filter using denoising AM.

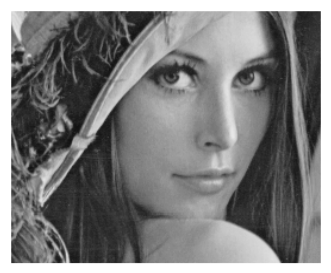

(a)



(c)

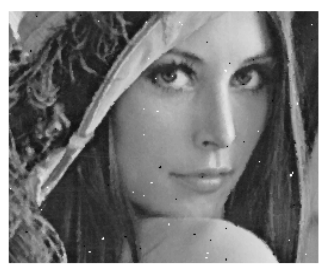

(b)



(d)
Fig. (5). Noise density of 0.8 local gray value images.

In the noise removal process, the adaptive window is the window change strategy to the noise detection mechanism using basically the same strategy described, except that the time necessary to determine whether the window scaling judgment using AM. Since the output value of the AM and the median value of the relevant window and its left neighbor, it can be assumed that when the non-filtering result AM extremum, then the window size can be used as the denoising window. After analysis can be found, that condition is more relaxed than the noise detection mechanism in the window changing conditions: When the value of the non-SM extremes, AM value nor extremes; but when SM is extreme value, since the value of AM by SM value judgment about its neighbors, AM value is also very likely not the extremes [8]. Therefore, it is possible to use this method of determining the window to get a smaller filter. We know by the preceding analysis, a smaller window, preserving image detail stronger, so the use of this strategy will be to achieve better results.

Literature [8] AM noise filtering algorithms are described in the introduction of a linear adaptive noise removal can be a good strategy, but for a high density of noise and image detail-rich treatment effect relative to other algorithm to be poor. The dynamic window policy, you can select the appropriate adaptive filtering window treatment, effective treatment of high-density noise and detail-rich images. In this paper designed for this defect adaptive median filtering method based on dynamic window (VAM) that is effective to improve on it.

\section{Algorithm 2: Noise Detection Algorithm}

Input: global image of the maximum value Gmax, the minimum value Gmin, the filter window is a maximum value Wmax, the pixel P $(a, b)$ and the gray value $G$, the threshold value $\mathrm{T}$.

$\mathrm{O}$ : pixel $\mathrm{P}(\mathrm{a}, \mathrm{b})$ is determined.

1. According to Algorithm 1, if the point $\mathrm{P}$ is non-noise point, the end, or else turns 2;

2. $\mathrm{P}$ as the center, sets the window size for the filter window w 3;

3. Calculate the current filter window, the filter algorithm uses the results of AM, denoted AM; if satisfied Gmin <AM $<$ Gmax, then go to 5 .

4. Sets the window size expands to $\mathrm{w}+2$; if $\mathrm{w}>\mathrm{Wmax}$, then goes to 5 , otherwise goes to 3 ;

5. To make a note of the current AM for VAM, as a result of the pixel filtering point $\mathrm{P}$ and $\mathrm{P}$ VAM updated pixel gray value after filtering.

\section{SIMULATION RESULTS AND ANALYSIS}

Adopt legend lena, barb filter simulation comparison. Which, lena image is more gentle, flat areas and more; and barb details of the plan are very rich, difficult to handle.

\subsection{Noise Analysis of Performance Testing Mechanism}

For the threshold value T1 2.1 mentioned different values, using VAM filter $512 * 512$ barb map denoising calculated SNR (PSNR), as shown in Fig. (6) plotted curve. Can be found, T1 values between 10-20 at the time, de-noising best, when T1 rising, along with de-noising effect of diminishing.

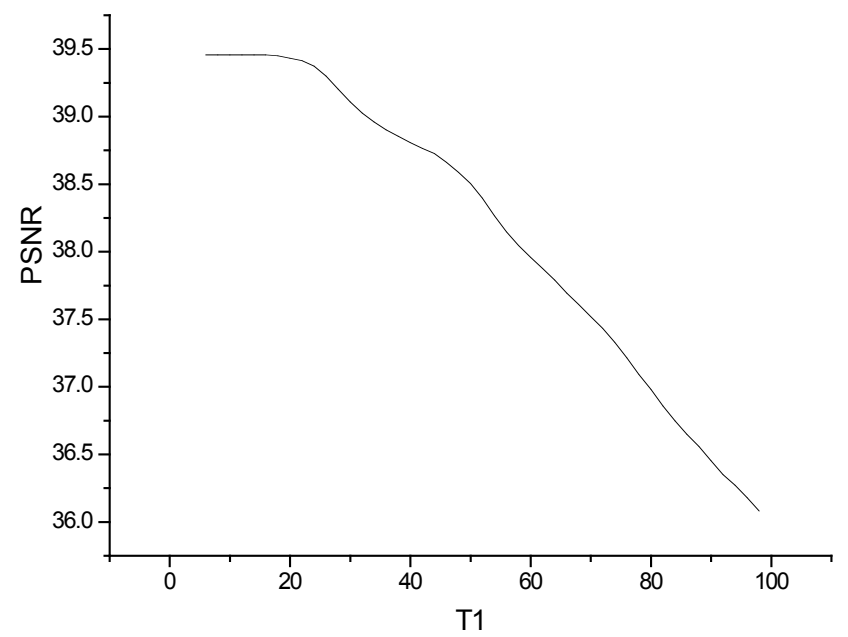

Fig. (6). different values of T1 VAM filtering the resulting signal to noise ratio. 
Table 1. Noise density noise detection mechanism found.

\begin{tabular}{|c|c|c|c|c|c|c|}
\hline \multirow{2}{*}{ Noise Density } & \multicolumn{3}{|c|}{ barb (512*512) } & \multicolumn{3}{c|}{ lena (512*512) } \\
\cline { 2 - 7 } & $\begin{array}{c}\text { Found that Noise } \\
\text { Points }\end{array}$ & $\begin{array}{c}\text { The Total Pixel } \\
\text { Image }\end{array}$ & $\begin{array}{c}\text { Detected Noise } \\
\text { Density }\end{array}$ & $\begin{array}{c}\text { Found that Noise } \\
\text { Points }\end{array}$ & $\begin{array}{c}\text { The Total Pixel } \\
\text { Image }\end{array}$ & $\begin{array}{c}\text { Detected Noise } \\
\text { Density }\end{array}$ \\
\hline \hline 0.2 & 52345 & 262144 & $19.97 \%$ & 52391 & 262144 & $19.99 \%$ \\
\hline 0.4 & 104991 & 262144 & $40.05 \%$ & 104702 & 262144 & $39.94 \%$ \\
\hline 0.6 & 157437 & 262144 & $60.06 \%$ & 157123 & 262144 & $59.94 \%$ \\
\hline 0.8 & 209804 & 262144 & $80.03 \%$ & 209631 & 262144 & $79.97 \%$ \\
\hline 0.9 & 235991 & 262144 & $90.02 \%$ & 236154 & 262144 & $90.09 \%$ \\
\hline
\end{tabular}

Table 2. Filtering method using various kinds of filters of different images in different SNR noise density after.

\begin{tabular}{|c|c|c|c|c|c|c|c|c|}
\hline & \multirow{2}{*}{ Image Name } & \multirow{2}{*}{$\begin{array}{c}\text { Filter } \\
\text { Window }\end{array}$} & \multicolumn{6}{|c|}{ PSNR } \\
\hline & & & Plus Noise Image & SM & CWM & TSM & $\mathbf{A M}$ & VAM \\
\hline \multirow{9}{*}{$\begin{array}{l}\text { Noise density } \\
\quad \text { of } 0.2\end{array}$} & \multirow{3}{*}{ lena } & $3 * 3$ & \multirow{3}{*}{34.0582} & 37.4139 & 40.2843 & 41.7077 & 42.2601 & \multirow{3}{*}{42.8497} \\
\hline & & $5 * 5$ & & 36.0394 & 37.144 & 39.6207 & 41.2163 & \\
\hline & & $9 * 9$ & & 34.7285 & 35.0574 & 37.5549 & 37.5103 & \\
\hline & \multirow{3}{*}{ barb } & $3 * 3$ & \multirow{3}{*}{34.0689} & 33.4826 & 35.7886 & 36.2758 & 36.4539 & \multirow{3}{*}{39.4598} \\
\hline & & $5 * 5$ & & 32.6353 & 33.3687 & 34.5618 & 34.7252 & \\
\hline & & $9 * 9$ & & 32.0913 & 32.3441 & 34.0033 & 33.9731 & \\
\hline & \multirow{3}{*}{ boat } & $3 * 3$ & \multirow{3}{*}{34.0966} & 36.0905 & 38.9921 & 40.2062 & 40.5004 & \multirow{3}{*}{41.5691} \\
\hline & & $5 * 5$ & & 34.8435 & 35.9231 & 38.2595 & 38.3394 & \\
\hline & & $9 * 9$ & & 33.6556 & 33.9739 & 36.5213 & 36.4122 & \\
\hline \multirow{9}{*}{$\begin{array}{l}\text { Noise density } \\
\quad \text { of } 0.4\end{array}$} & \multirow{3}{*}{ lena } & $3 * 3$ & \multirow{3}{*}{31.061} & 35.0758 & 37.376 & 37.3803 & 37.36 & \multirow{3}{*}{38.912} \\
\hline & & $5 * 5$ & & 35.2476 & 36.3477 & 37.4348 & 37.6484 & \\
\hline & & $9 * 9$ & & 34.3614 & 34.7378 & 36.2427 & 36.2736 & \\
\hline & \multirow{3}{*}{ barb } & $3 * 3$ & \multirow{3}{*}{31.0276} & 32.4786 & 34.5919 & 34.3919 & 34.3538 & \multirow{3}{*}{36.1053} \\
\hline & & $5 * 5$ & & 32.3468 & 33.1875 & 33.7383 & 33.8441 & \\
\hline & & $9 * 9$ & & 31.9389 & 32.2315 & 33.2873 & 33.2817 & \\
\hline & \multirow{3}{*}{ boat } & $3 * 3$ & \multirow{3}{*}{31.0568} & 34.1081 & 36.4528 & 36.3512 & 36.265 & \multirow{3}{*}{37.6799} \\
\hline & & $5 * 5$ & & 34.1699 & 35.274 & 36.2101 & 36.2789 & \\
\hline & & $9 * 9$ & & 33.4419 & 33.7907 & 35.2856 & 35.206 & \\
\hline
\end{tabular}

In the case where the value of $\mathrm{T} 115$, respectively, and the barb lena image by adding a certain density of the image noise, then the noise detection operation, which counts the number of noises and the noise density is found as shown in Table $\mathbf{1}$. As it can be seen, the noise detection mechanism works well, the noise detection result and the actual density error is small.

\subsection{VAM Filter de-Noising Effect Analysis}

Table 2 shows the noise density of 0.2 and 0.4 , lena, barb and boat three figures denoising results using different filter- ing algorithms. As it can be seen from the figure, AM filter algorithm has a good relative to other improvements, but the results of the texture of the resulting complex boat FIG deviations than other algorithms. The VAM proposed algorithm is different for the various features of the image have a better denoising effect, which is due to VAM while retaining the advantages of AM gentle image processing, while insufficient to overcome the AM when rich in detail, and to strengthen the retention capacity and the ability to image details denoising image. 

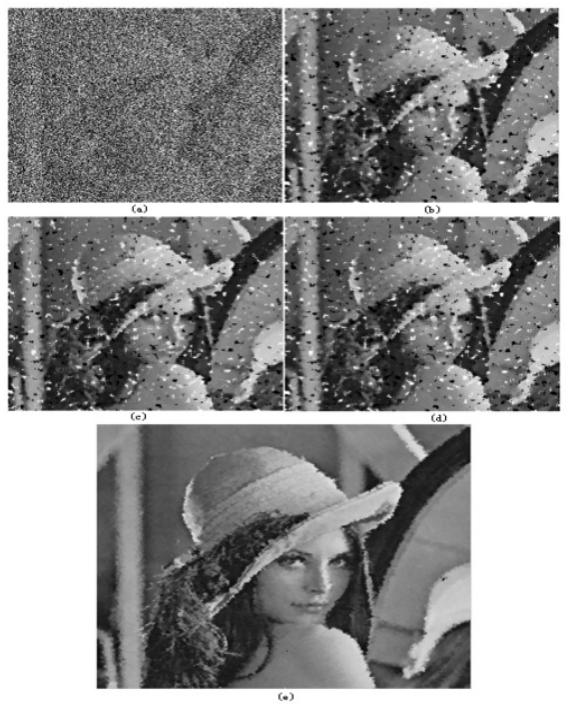

Fig. (6). Noise density of 0.8 lena denoising results.

VAM in order to verify the adaptive window policy than the fixed window policy in dealing with the advantages of a high density of noise on the image, Fig. (7) shows the image processing conditions for lena containing 80\% noise. Wherein the drawing (b), (c), (d) are filtered result WM, TSM, AM and AVM's. As can be seen, with adaptive resize the window function VAM algorithm still has a strong processing capacity for high-density noise, noise can be accurately filtered out, and the image detail and edge information retention is good.

\section{ENDING}

This paper presents an adaptive median filter noise detection method (AVM) based. The new algorithm uses reasonable noise detection mechanisms window adaptive strategy designed to achieve accurate and efficient point of distinction between noise and non-noise points, thereby protecting the details of the image edge information. After comparing the experimental simulations, noise filtering algorithms described in this article with respect to other typical algorithms, and details on the noise processing significantly improved protection for high density image noise, the more obvious advantages.

\section{CONFLICT OF INTEREST}

The authors confirm that this article content has no conflict of interest.

\section{ACKNOWLEDGEMENTS}

Declared none.

\section{REFERENCES}

[1] T. Lu, "digital image processing", CA: Beijing, pp. 85-97, 2007

[2] D. R. K. Brownrigg, "The weighted median filter," Communications of the ACM, vol. 27, pp. 807-818, Aug. 2007

[3] S. J. Ko and Y.H Lee, "Center weighted median filters and their applications to image enhancement," IEEE Transactions on Circuits and Systems, vol. ED-38, pp. 984-993, Sep. 2008.

[4] T. Chen, K. Ma, and L. Chen, "Tri-State. Median Filter for Image Deonsing," IEEE Transactions on Image Processing, vol. ED-8, pp. 1834-1838, Dec. 2007.

[5] H. Hwang and R. A. Haddad, "Adaptive median filters: new algorithm and results," IEEE Transaction on Image Processing, vol ED-4, pp. 499-502, Apr. 2006.

[6] X. H. YANG, and P. S. TOH, "Adaptive fuzzy multilevel median filter," IEEE Transaction on Image Processing, vol. ED-4, pp. 680682, May. 2008.

[7] H. L. ENG, and K. K. MA, "Noise adaptive soft-switching median filter," IEEE Transaction on Image Processing, vol. ED-10, pp. 242-251, Feb. 2009.

[8] C.C. Chang, J.Y. Hsiao, "Chih-Ping Hsieh. An Adaptive Median Filter for Image Denoising," IEEE Second International Symposium on Intelligent Information Technology Application, Shanghai, pp. 346-350, 2008

[9] R. Chang, and X. Mu, "adaptive filtering algorithm in the mean value of," Based Computer Engineering and Design, vol. ED-29, pp. 4257-4259, Aug. 2008

[10] M. Wang, J. Cheng, F. Zhang, "An improved adaptive median filtering algorithm," Microcomputer Information, vol. ED-26, pp. 109-110, Apr. 2010. 